\title{
Solar Dynamical Processes I
}

\author{
Ashish Mishra $^{1 *}$, Mukul Kumar ${ }^{1,2^{*}}$ \\ ${ }^{1}$ Department of Physics, Indian Institute of Science Education and Research Bhopal, India. \\ ${ }^{2}$ State Key Laboratory of Space Weather, National Space Science Center, \\ Chinese Academy of Sciences, Beijing, China.
}

*Corresponding Author email:

1 ashu.mishra274@gmail.com

2 mk.cas@yahoo.com

Article Histary

Received: 18 December 2017

Revised: 15 January 2018

Accepted: 27 January 2018

Published: 30 January 2018

Student(s)

- $\quad$ Ashish Mishra

Academic Year: 2015-16, $2^{\text {nd }}$ Semester

Course Level: Bachelor

Course Name: BS-MS dual degree program

Course year: $4^{\text {th }}$ Year $/ 8^{\text {th }}$ Semester

Mentar(s)

- Mukul Kumar

\section{A B S T RA C T}

The article gives a concise overview of solar dynamical processes and their impacts on the space weather. This article is based on the observational and theoretical developments made during last few decades. The article begins with a brief discussion of the Sun and the solar interior, from the core to the solar corona. We discuss the solar magnetic field and provide some basic understanding of the solar dynamo model. The solar dynamical processes, the transient as well as the gradual, are the manifestations of the Sun's magnetic field. Magnetic reconnection, as well as submergence and emergence of magnetic flux tubes, plays an important role in the solar activities. This article tries to cover a range of dynamical processes, including sunspots, solar prominences and bright points. We also discussed various models of the dynamical processes along with their properties and effect on other activities occurring on the Sun.

Keywords: Sun, Solar dynamical process, Solar magnetic field, Solar dynamo, Sunspots, Prominence, Bright Points, Coronal mass ejection, solar flares, coronal holes

\section{Introduction}

\subsection{The Sun}

Sun is a ball of gas and plasma, rotates on its own axis as well as around the centre of mass of the Milky Way Galaxy. The Sun is categorized as a G-type main sequence star whose surface temperature is in the range of $5500 \mathrm{~K}$ to $6000 \mathrm{~K}$. The sun is considered as an almost perfect sphere with homogeneous composition. The mean distance between the Sun and the Earth is $1.496 \times 10^{11} \mathrm{~m}$, also known as 1 Astronomical Unit (AU), [1] - [7]. A few physical properties of the Sun are illustrated in table 1. Because the Sun's shape is symmetric and almost spherical, it also follows the hydrostatic and mechanical equilibrium (which is valid for spherically symmetric stars) in which the pressure gradient balances the gravitational forces (The hydrostatic and mechanical equilibrium) [9].

$$
\begin{gathered}
\frac{d P(r)}{d r}=-\frac{G m(r)}{r^{2}} \rho(r) \\
\frac{d m(r)}{d r}=4 \pi r^{2} \rho(r)
\end{gathered}
$$

where $\mathrm{P}(\mathrm{r})$ is the pressure, $\varrho(r)$ is the density and $\mathrm{m}(\mathrm{r})$ is the mass within a radius of $\mathrm{r}$. 
Table 1: Some physical parameters of the Sun [9]

\begin{tabular}{|l|c|}
\hline Property & Value \\
\hline Mass & $(1.9891 \pm 0.004) 10^{30} \mathrm{~kg}$ \\
\hline Equatorial Radius & $(6.95991756) 10^{8} \mathrm{~m}$ \\
\hline Polar Radius & $(6.95984388) 10^{8} \mathrm{~m}$ \\
\hline Mean Density & $1409 \mathrm{~kg} \mathrm{~m}^{-3}$ \\
\hline Age & $4.52 \pm 0.4 \mathrm{Gyr}$ \\
\hline Gravitational acceleration & $274.0 \mathrm{~m} \mathrm{~s}^{-2}$ \\
\hline Luminosity & $(3.846 \pm 0.05) 10^{26} \mathrm{~W}$ \\
\hline Gravitational acceleration & $274.0 \mathrm{~m} \mathrm{~s}^{-2}$ \\
\hline
\end{tabular}

The most abundant constituent of the Sun is Hydrogen (More than 75\%) while Helium is the next most abundant element of the Sun. Six most abundant elements in the Sun are hydrogen, helium, carbon, nitrogen, oxygen and neon [10], [11]. The nuclear reaction taking place in Sun accounts for energy released into the interplanetary space (see Table 2). The change in Earth's climate and the weather is a result of various solar activities and dynamical processes going on in the Sun.

Table 2: Nuclear processes in the core of the Sun [12]

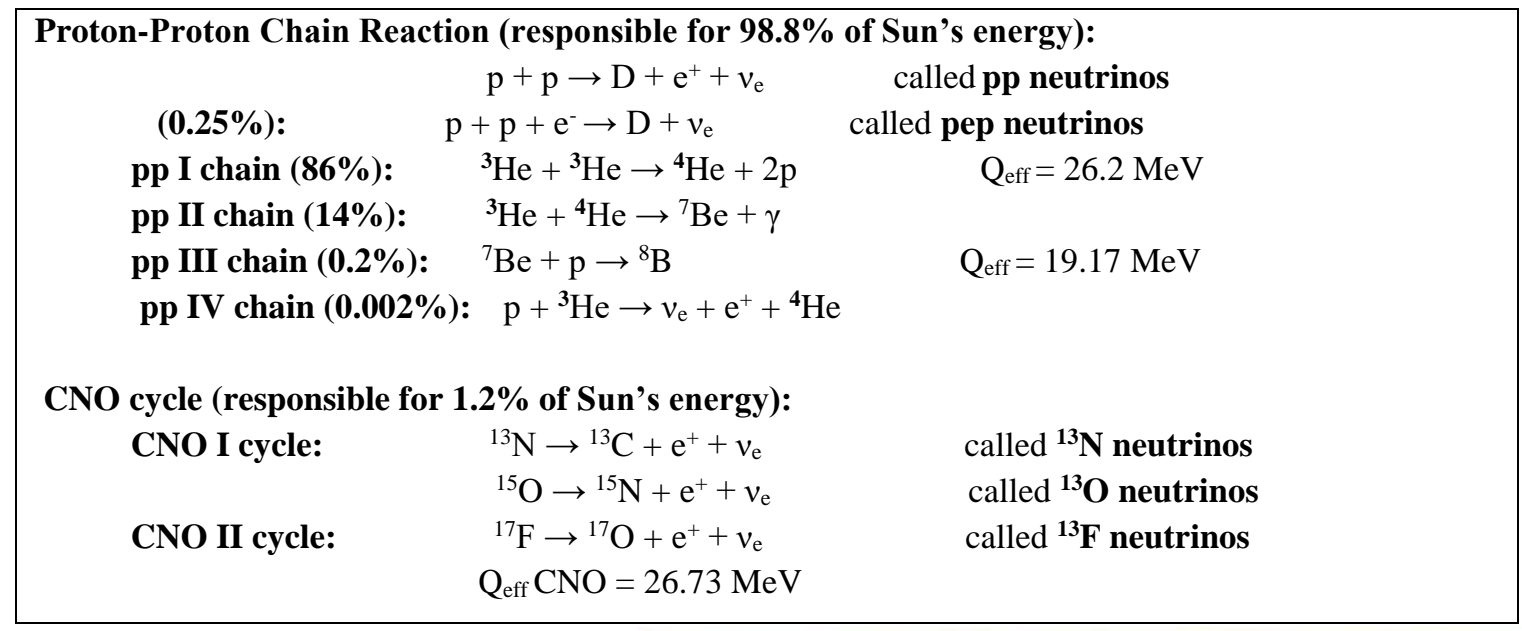

\subsubsection{Structure of the Sun}

The solar structure has always been an intriguing area of research as the solar interior cannot be directly observed. Investigation of the solar structure with stellar equations and assumptions, solar oscillations (helioseismology), and neutrino observations have helped to develop the "standard model" of the Sun [1], [5]. According to the standard model, the Sun and its atmosphere (heliosphere) can be divided into different layers (see figure 1). The helioseismological developments aided to the reasonable interior structure of the Sun [12] - [17].

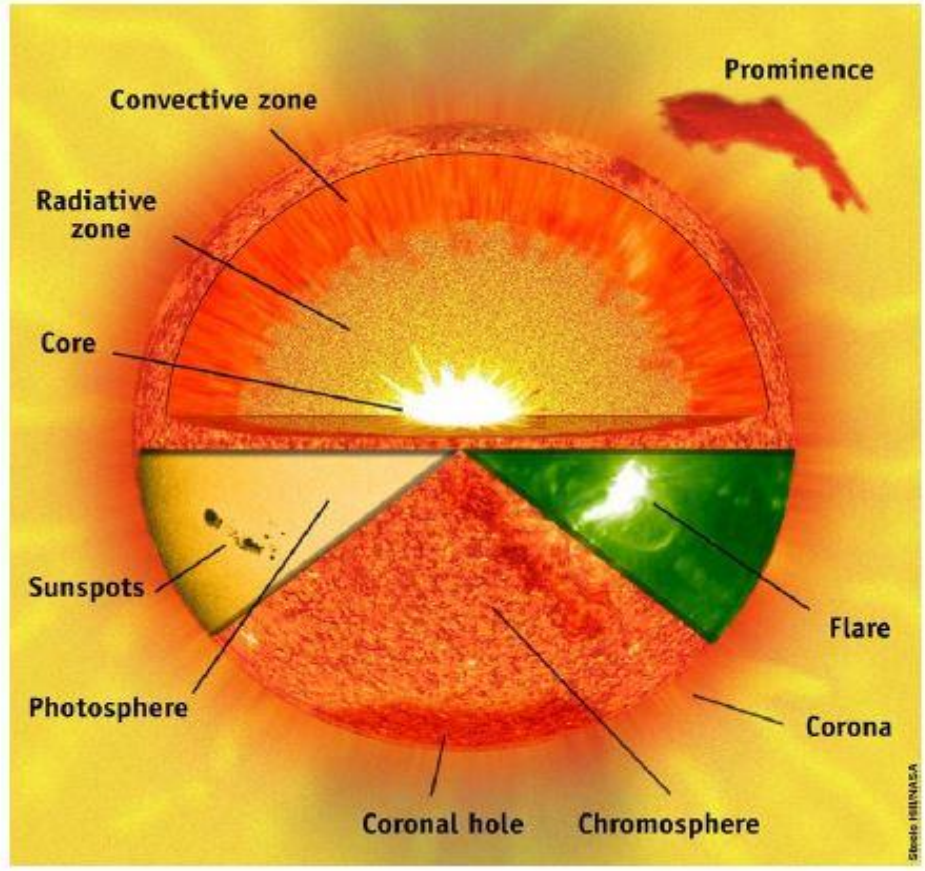

Figure 1: Structure of the Sun depicting its different layers [18] 
Mishra et al., Adv. J. Grad. Res.; Vol. 3 Issue 1, pp: 47-61, January 2018

The Core: Core, the innermost and most dense part of Sun, out to about $15-20 \%$ of the solar radii and often referred as the powerhouse of the Sun, where nuclear fusion takes place. Energy production by fusion occurs at a temperature of about $10^{7} \mathrm{~K}$ by a stepwise proton-proton $(\mathrm{p}-\mathrm{p})$ chain reaction. This reaction leads to a formation of Helium from hydrogen ions. During the reaction, various types of high energy radiations and particles are also emitted from the Sun's core [12], [19]. Another cycle (CNO cycle) is also responsible for energy production on the Sun see table 2. Haubold and Mathai [19] studied the solar neutrino fluxes and suggested that solar nuclear reactions are stabilized by the gravitational force of Sun.

Radiative Zone: The layer next to the core of the Sun is radiative zone, ranges from 0.15 to 0.7 solar radii. The energy generated in the core is transported to radiative zone by thermal radiation (photons). There is a fall in temperature gradient outwards the Sun's core along with the transfer of energy by radiated photons. The density of the radiative zone is very high, and so, its mean free path is very small. A photon generated at the core of Sun may take millions of years to cross the radiative zone.

Convection Zone: This zone is extended outwards from the Sun's radiative zone up to the photosphere, i.e., from 0.7 to 1 solar radii. The temperature of convective zone is less than that of radiative zone. It transfers the energy obtained from radiative zone to photosphere by the process of convection and hence named as convection zone. Observations and Study of helioseismological data suggest that the radiative zone of the Sun (along with the core) rotates uniformly while the convective zone rotates non-uniformly [16], [17]. As a result, there is strong rotational shear between radiative and convective zones and this shear leads to a formation of a thin layer known as "tachocline".

Tachocline: It is often considered as a separate layer (less than 0.1 solar radii) sandwiched between radiative zone and convective zone. It is considered to be responsible for the generation of the solar magnetic field. As discussed previously, the interior of Sun does not rotate in the same way as does its surface. However, it is believed that the inner regions of the Sun including the Sun's core and radiative zone rotate more like a solid body [5], [6], [15] - [17], while the outer part of the Sun (from the convective zone) rotates at different rate that varies with latitude. The boundary between radiative zone and the convection zone produces a shear due to a different rate of rotation. This shear gives rise to a very thin layer called the 'tachocline'. It is believed that tachocline is the site of the origin of Sun's magnetic field.

Photosphere: The outermost surface of Sun is called photosphere. The temperature of the photosphere lies in the range of 5800-6000 K. The regions with considerably lower temperature in the photosphere, visible as dark spots, are called sunspots with the temperature in a range of $3000-4500 \mathrm{~K}$. The highresolution instruments, let us observe the granular structure of photosphere (see Figure 2). Granulation [3], [4], [20] [21] in the photosphere occurs due to the convective currents which carry a large amount of energy and flows from convective zone to the photosphere.

Chromosphere: The layer above the photosphere, called chromosphere which is approximately 1500 kilometres thick. This layer has a temperature greater than that of the photosphere which lies in the range of $10000-12000 \mathrm{~K}$. It is the site of many dynamical processes such as prominence, coronal loop etc. The region sandwiched between chromosphere and corona is called transition zone or transition region, and in this region, there is a rapid increase in the temperature to coronal values [22].

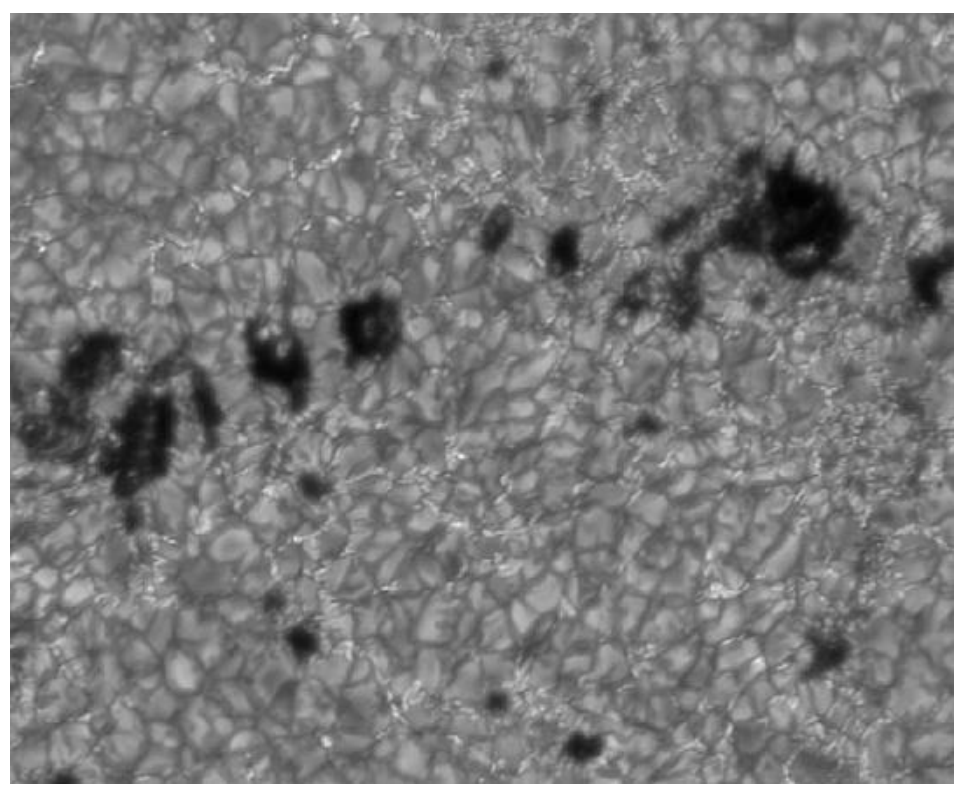

Figure 2: Photosphere, granules visible along with sunspots [4] 
Solar Dynamical Processes I

Corona: The outermost layer of the heliosphere is called corona. The temperature of corona vary in the range of $10^{6}-10^{7} \mathrm{~K}$. Corona is visible by naked eyes during the time of the total solar eclipse. This drastic increase in the temperature from the photosphere to corona is still a fundamental problem in solar research.

\subsubsection{Solar Magnetic Field}

General magnetic field of the Sun is bipolar in nature. It is more concentrated at poles and has average intensity approximately equal to 1 Gauss [24]. It has been observed and studied in detail [23] - [31]. General potential field model [23] suggests loops of magnetic field all over the surface of the Sun (see figure 3). The magnetic field of Sun is very dynamic and it keeps on changing with time. It is believed that magnetic field of the Sun is a result of the motion of plasma in tachocline.

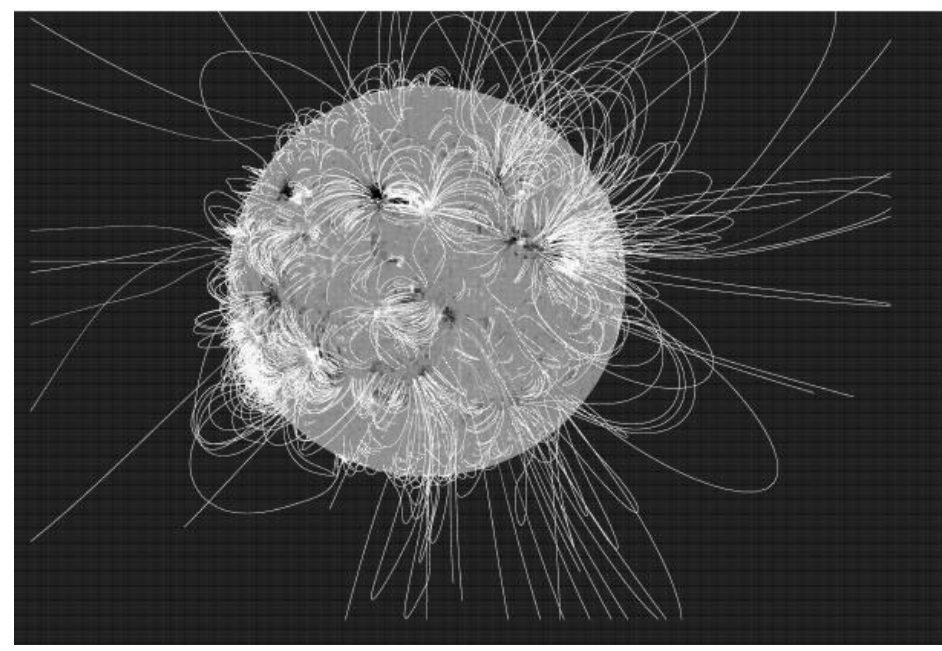

Figure 3: Global Potential field model [23]

One of the most important properties of Sun is its differential rotation. Just like Earth, Sun also rotates on its axis. But unlike the Earth, different regions of the Sun's rotate with different speed i.e., Sun's equator rotates at a faster rate than its pole. This difference in the rotation rates of the Sun is called the differential rotation. Differential rotation is apparently the driver of the 11year sunspot cycle and the associated 22year solar cycle. There have been various dynamo models have been put forward [32] - [37]. Eugene N. Parker [37] showed that magnetic field can be regenerated by rotating sphere of conducting fluid. He suggested that if the cyclone is present in the convective zone, then their rotation will be strongest near the pole and approximately vanish near the equator. As we have already discussed the differential rotation, shearing due to this will be stronger at the equator as compared to that of poles because of faster rotation of the equator (see figure 4). Parker [37] suggested that there will be toroidal magnetic field produced due to shearing and it will be strongest at the equator and poloidal magnetic field will be produced by the cyclonic motion which will be strongest at the poles.

As we have developed an understanding of the solar magnetic field, we can now have a broader look at other solar activities. In this article, our motive is to understand solar dynamical processes in detail
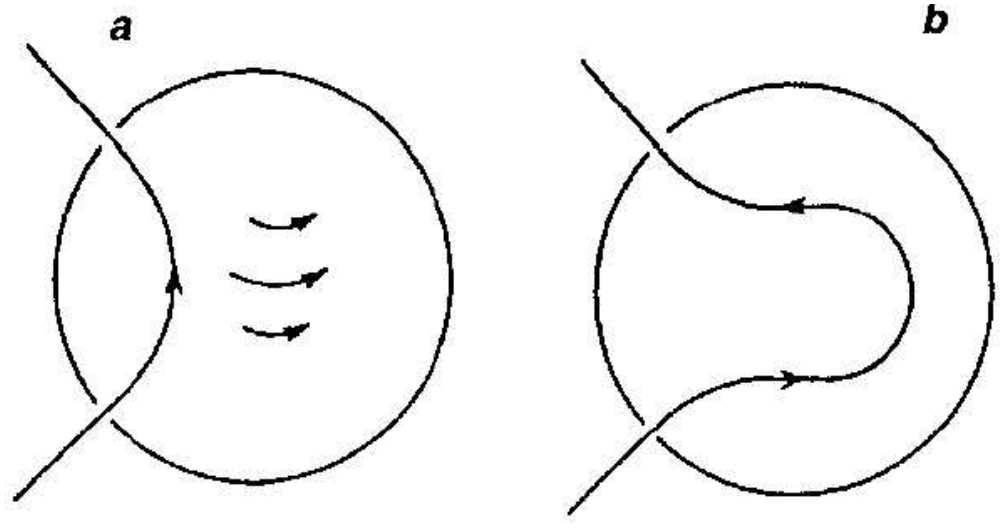

Figure 4: Production of magnetic field underneath Sun's surface. a. Poloidal magnetic field. $b$. Stretch in magnetic field due to faster rotation near equator [33] 
Mishra et al., Adv. J. Grad. Res.; Vol. 3 Issue 1, pp: 47-61, January 2018

and their effect on space weather. Events like prominence and filament eruptions can trigger CMEs and flares which can lead to very strong geomagnetic storms on Earth which could have hazardous consequences.

\section{Solar Dynamical Processes}

Solar dynamical process refers to the process and phenomenon occurring in the Sun's atmosphere and evolving or changing very fast. These dynamical processes take place all over the magnetic environment of the Sun throughout the solar cycle. Depending upon the time of solar cycle (solar minimum or maximum), the dynamical process also varies in their occurrence, strength and composition. During the time of high solar activity, dynamical processes evolve or change faster, which leads to, often eruptive solar events like flares, coronal mass ejections, prominence eruption etc., and the sun is often referred to as Active Sun. During the time of low solar activity, dynamical processes are often seeming to be quasi-static, and the eruptive phenomenon is very low in number. The Sun during that time is called Quiet Sun, and seemingly stable prominence structures are found in the chromosphere. In the active regions, solar magnetic field changes very fast, leading to many dynamical processes such as formation and eruption of prominences, sunspots, solar energetic particles, solar winds, coronal mass ejections, solar flares etc. and these dynamical processes are the main drivers of space weather [38].

\subsection{Sunspots}

There are many magnetic activities that occur on Sun. Sun's magnetic field is one of the most inconsistent phenomena on the Sun with a definite cycle. One of the important phenomena on the photosphere of the Sun due to its magnetic field is sunspot. Sunspots are the dark regions on Sun's photosphere which have highly converged and concentrated the magnetic field. This highly concentrated magnetic field restrict the convection to take place due to which there is a significant decrease in temperature of these regions or spots. In comparison to the temperature of photosphere (5800 - $6000 \mathrm{~K})$, the temperature of a sunspot is significantly lower (ranging from 3000 to $4500 \mathrm{~K}$ ). Due to this low temperature, the luminance of these spots is less than that of the surrounding photosphere, so these regions appear dark and are called sunspots. Sunspots can be seen with naked eyes. Many evidence of sunspot observations has been recorded in the past. They have been observed by Galileo, Kepler etc. [39]. One of the most important features of a sunspot was observed by Heinrich Schwabe from his observation from 1826 to 1843. He noticed that number of sunspots varied regularly with a period of 11 years. R.C. Carrington [40], [41] found that with the course of time, sunspots latitude decreases i.e., sunspots move from higher latitude to lower latitude towards the equator during a cycle. This observation later came to be known as Sporer's law.

In 1908, G.E. Hale [42] studied the Zeeman effect in sunspots and through his observations of photographs of sunspots, he suggested that all sunspots are vortices. He further linked it with the existence of a magnetic field in sunspots. Further, he suggested the hypothesis that the sunspot is a vortex in which electrified particles, produced by ionization in the solar atmosphere are whirled at high velocity and this phenomenon might give rise to the magnetic field in sunspots regarded as electric vortices [43].

Hale et al. [43] in their observation of magnetic polarity of sunspot suggested that most of the sunspots are binary groups in which one sunspot has opposite magnetic polarity than that of other, while the unipolar spots exhibited the characteristics of bipolar groups. They also linked the magnetic activity of Sun and sunspots cycle. There are many observational properties of sunspots were studied from time to time to understand the magnetic field of the Sun. It was readily thought that the sunspots were the result of differential rotation of the Sun i.e., the magnetic energy of the sunspot was provided by the differential rotation of the Sun.

Cowling [44] studied the growth and decay of sunspots in detail. He suggested that growth and decay of a sunspot may be due to the differential rotation of the Sun. He concluded that electromagnetic phenomenon alone cannot be the deciding factor in the growth and decay of the magnetic field in the sunspots and differential motion plays an important role in the same. Cowling [44] also suggested two possibilities of 
Solar Dynamical Processes I

growth and decay of sunspots (as stated in his paper), "One possibility is that line of force of Sun's general magnetic field are bunched together into a spot as a result of mass motion and the second possibility he mentioned is that a field exists below the surface, line of force running as 'girdles' round the Sun's axis, and a pair of spots arises when part of this field is convected into the surface."

Parker [37] suggested that due to hydromagnetic motions in the convective zone of the Sun, a poloidal magnetic field is produced which is stronger at the poles than the equator, whereas due to differential rotation, a toroidal field is produced which is also stronger at the equator than the poles. He suggested that sunspots are produced due to the magnetic buoyancy of toroidal magnetic field and there should be a number of sunspots in lower latitudes. This result of finding sunspots at lower latitudes was consistent with the studies of Hale et.al. [43].

According to Hannes Alfven [45], the differential rotation alone cannot be a source of production of sunspots. He argued that the energy produced by differential rotation of Sun is not enough to empower the sunspots. He further suggested that magnetic energy of the sunspots comes from the nuclear reaction in the solar core. He assumed that heat produced in the Sun's core is converted into mechanical and electromagnetic energy in the convective zone and can be helpful as a source of sunspot's energy.

Babcock [25] showed in his model that differential rotation twists the lines of force and provide energy to magnetic fields and thus, the differential rotation can lead to the eruption of the magnetic field through the solar surface and can eventually form a photosphere. R.B. Leighton [28] supported the work of Babcock [25] but Leighton suggested that differential rotation does not always amplify the magnetic field rather it can also reduce the magnetic field. He suggested that the amplification of magnetic field by the differential rotation lasts only till the formation of sunspots and after the formation of a sufficient number of sunspots the magnetic field starts reducing by the effect of differential rotation [28].

H. Zirin [31] suggested that field line reconnection take place to keep the field in lower energy state. He also suggested that when near the sunspot bipolar flux emerges or erupts then to be in lower energy state, magnetic reconnection occurs. This leads to the release of energy and a flare eruption can take place.

Meyer et al. [46] suggested that strong converging super granular flows of the magnetic field are required to form and stabilize the sunspot. They suggested that strong and concentrated magnetic field inhibit the convection and equilibrium are achieved. This is the main reason for a low temperature of sunspots. Their theory was based on the assumption that sunspots are axially symmetric and surrounded by an annular convection cell. They also suggested that upward motion of magnetic flux from deep convective zones leads to the formation of active regions. This flux (on reaching the photosphere) is concentrated by super granular convection and forms a sunspot.

As we already discussed, sunspots can be observed and identified on the basis of their relative brightness with respect to the surrounding photosphere. sunspots are made up of a central and darker part called the umbra, which is surrounded by the less dark part called the penumbra (see figure 5). The magnetic field strength is higher in the central dark part i.e., umbra and the field lines are very much concentrated and vertical while penumbra have relatively weaker magnetic field and field lines are more inclined to the surface normal i.e., magnetic field in umbra is homogeneous whereas magnetic

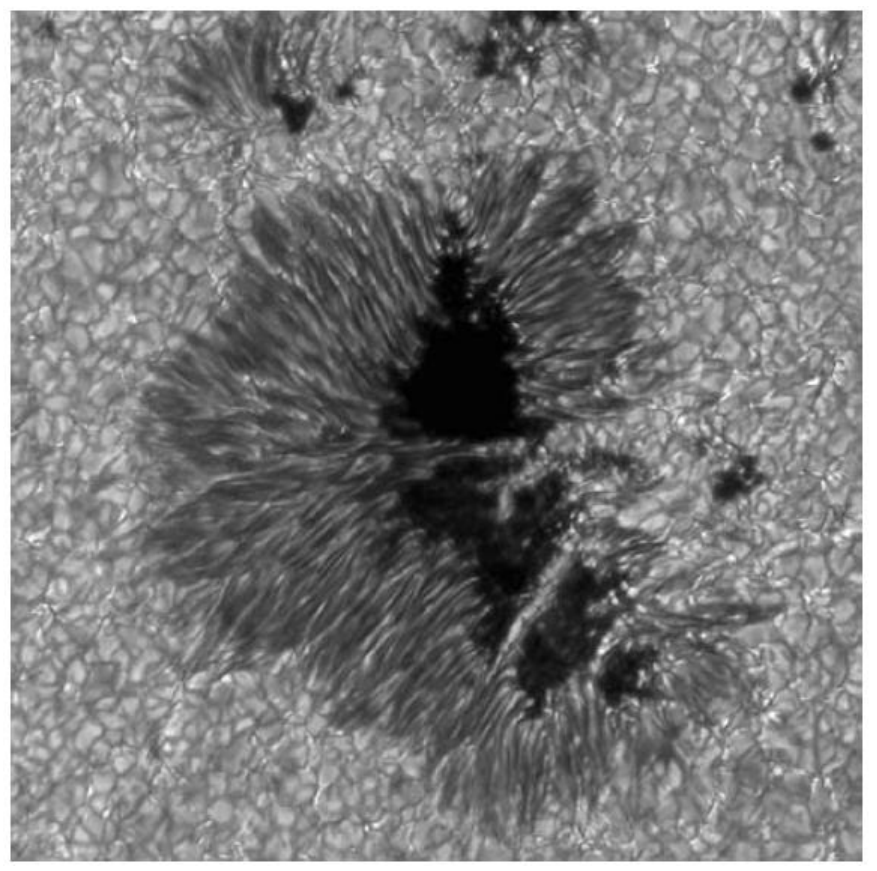

Figure 5: Sunspot with faculae structure having Umbra and Penumbra. Here granulation of the photosphere can also be seen [47] 
Mishra et al., Adv. J. Grad. Res.; Vol. 3 Issue 1, pp: 47-61, January 2018

field in penumbra is non-homogeneous and points radially outwards. There is a large variation in the size of sunspots and can vary up to an order of $10^{4} \mathrm{~km}$ or more. Larger sunspots can be seen by naked eyes also. Smaller sunspots are common on Sun's photosphere.

Umbra is the coolest part of the spot with a temperature range of 3000 to $4500 \mathrm{~K}$ and penumbra is hotter with the temperature range of about 5000 to $5500 \mathrm{~K}$. Observations [47] - [49] from better resolution instruments have revealed a fine structure with different parts of umbra and penumbra too. There appear bright dot-like structures inside the umbra called the umbral dot with few darkest part of the umbra called dark nucleus. The dark nucleus is assumed to be structureless. Between the umbra, there is a bit brighter region than the dark nucleus and is called diffuse background. The inner region of penumbra has a bright thread-like or filamentous structure known as penumbral grains. The penumbral grains are dynamic and radially move in the penumbra.

Sunspots have a dynamic structure. Some of the dynamical features of sunspot include meridional motion, sunspot cycle, the motion of spots relative to each other, flux emergence phenomenon etc. These motions are very important as they determine various dynamical process on Sun. Sunspots and their dynamic phenomenon have been studied in detail for over a long period of time. Its effect on climate and weather on Earth has also been studied, examined and understood in detail [47], [50], [51]. Researchers have analysed various databases related to sunspot dynamics, their number and climate variation [3], [9], [47], [50], [51]. It has been observed that there were various periods when the number of sunspots was very less, for example, the Maunder minimum (1624 - 1715) and Dalton minimum (1795-1820), see figure 6. Incidentally, it has been noted that the "Little Ice Age (1450-1820)" (colder periods) coincided with these noted sunspots minimum.

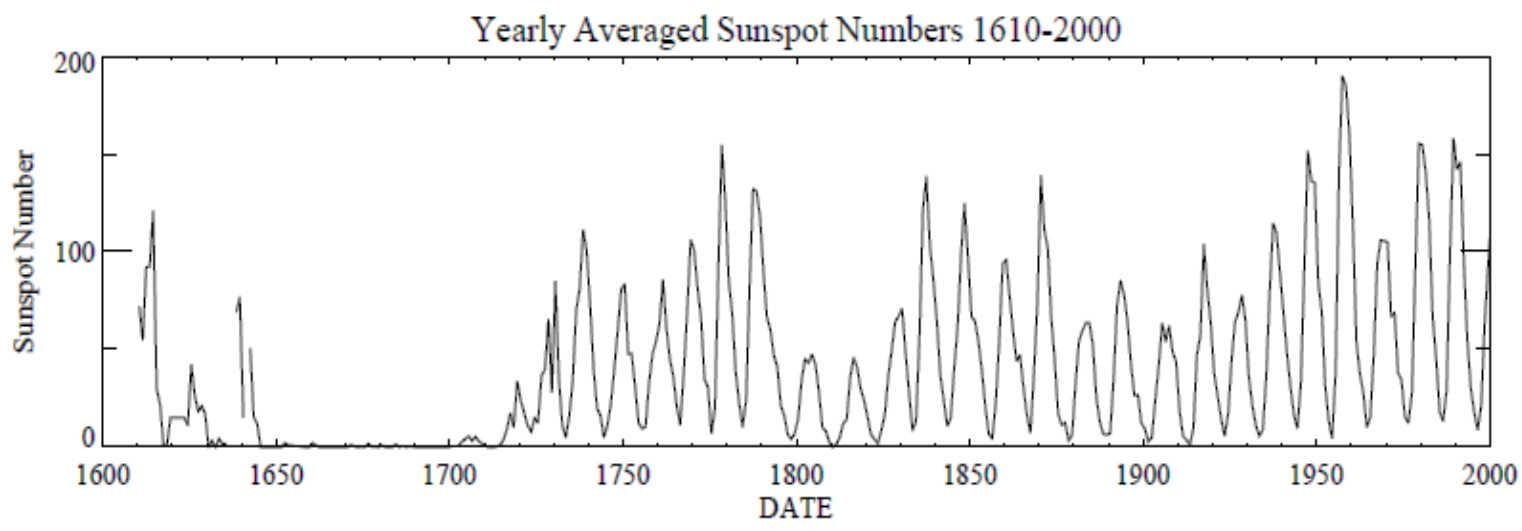

Figure 6: The monthly number of sunspots plotted against time for the period 1600-2000 [33]

A decline in geomagnetic activities during solar minimum has been observed by scientists (e.g., [52]. B. Kirov et. al. [52] concluded that during solar minimum, changes in the parameters of high-speed solar wind, and change in thickness of heliospheric current sheet are the important factors for geomagnetic activities. Geerts and Linacre [50] in their work clearly stated that "intuitively one may assume that the total irradiance would decrease as the number of (optically dark) sunspots increase. However, direct satellite measurements of the irradiance have shown just opposite to be the case. This means that more sunspots deliver more energy to the atmosphere so that global temperature should rise." It is also believed that there is a relation between the sunspot activity and increase of Earth's temperature.

It has been observed that the sunspots mostly occur in solar active regions and more sunspots are observed during the periods of increased magnetic activity. They are the source of various kind of oscillations (e.g., see [53], [54] and citations therein) and can be linked to other solar dynamical processes. Sych et. al (2015) studied the waves emanating from the sunspots and their relation with solar flares [55]. Their study was based on SDO/AIA data and they found that in the region of sunspots, waves through magnetic channel 
Solar Dynamical Processes I

played an important role in releasing solar flare energy. They further proposed that these amplified waves triggered the solar flare.

During the periods of high magnetic events, energetic particles and cosmic rays are emitted out of the Sun and they mix with the solar wind to propagate in the interplanetary space. This can lead to various events (like auroras) and geomagnetic activities on Earth. Sunspots play a very crucial role in our understanding of Sun's magnetic field and the phenomenon which directly affects the human life on Earth.

\subsection{Prominences}

The solar prominence is a large arch-type structure of gas extended into the much hotter outer atmosphere of the sun (in the corona). When observed above the solar limb, prominences are anchored in the photosphere and have large magnetic structures. Plasma confined in this structure in relatively cool $\left(\sim 10^{4} \mathrm{~K}\right)$ than the surrounding corona $\left(\sim 10^{6} \mathrm{~K}\right)$, so the prominence is approximately 100 times cooler than the surrounding corona. Recently, Solar speculated the heating of solar prominences by Alfven waves and showed that heating by Alfven waves is small but significant for energy balance [56].

The prominences are the dense objects. Their density is even higher than the corona and varies in the range of $10^{9} \mathrm{~cm}^{-3}$ to $10^{11} \mathrm{~cm}^{-3}$ with very low gas pressure. When prominence is observed above the chromosphere (i.e., against solar disk), they appear dark threads like structures held in the chromosphere and are called filaments. Prominence (or filaments) appear dark because they are cooler than their surroundings and their illuminance is lesser as in the case of sunspots.

Prominence is normally located above the magnetic polarity inversion line (PIL) of the photosphere. PILs are formed all over the photospheric region depending upon its magnetic configuration. So, the prominences are also found all over the Sun's surface i.e., in the active regions as well as quiet regions.

On the basis of their location on the Sun, prominences are generally classified into two types: active region prominence and quiet region prominence. Active regions prominences (called active prominence) are generally shorter and less stable than the prominence in the quiet region (called quiescent prominence). The prominences can be categorized on the basis of their origin, motion, structure etc. e.g. [57].

Solar prominences are observed to have a mass of roughly of the order of $10^{16} \mathrm{~g}$, with the uncertainty that can lie with the limits of $10^{4} \mathrm{~g}$ to $10^{17} \mathrm{~g}$ [58], [59]. Low et. al., [59] studied the role of the mass of prominence as a source of energy storage and suggested that it is necessary to hold the unanchored part of the magnetic field of prominence in equilibrium against the field's natural tendency to self-expel out of the atmosphere. It is evident from the observations that prominences can be found in highly complex topologies while the quiescent prominences are usually having simple magnetic topology.

The magnetic field of solar prominences plays a vital role in their structure and stability [60] - [70]. The magnetic field in the prominence is 'frozen' and helps to confine a large amount of plasma within the prominence. The magnetic field is almost poloidal, normally like a magnetic field above PIL. It is highly sheared at the top of the arcade (nearly horizontal). Prominence may also contain azimuthal magnetic field component. Correll and Roberts [63] studied the prominence and calculated the magnetic field strength with a lower limit of nearly 5 gausses and predicted that lower limit ( 5 gausses) may be higher. Further, in 1961 Zirin and Severny [64] calculated the magnetic field strength in solar prominence and suggested that in the active region, prominences can have strong (up to the range of 200 gausses) magnetic field while the magnetic field of quiescent prominence is much weaker with a range of about 25-50 gauss [64]. Leroy et. al., [66] based on their study on Hanle effect derived the strength of magnetic field in a prominence. They concluded that average field strength in a prominence is about 8 gauss and the field strength vertical gradient is $0.5 \times 10^{-4}$ gauss $\mathrm{Km}^{-1}$. Kuckein et. al., [65] suggested that active region prominences have a strong transverse magnetic field strength which is higher 500 gauss but lower than 1-kilogauss.

The structure of prominence has been studied in detail for past few decades and different models regarding their stability have been proposed. It has been found that prominence formation requires formation of magnetic structure (like the magnetic arcade) and support for holding the structure (carrying flux) in a stable form. They are mostly formed near the PIL where flux transportation is easy e.g. see [67] - [70]. 


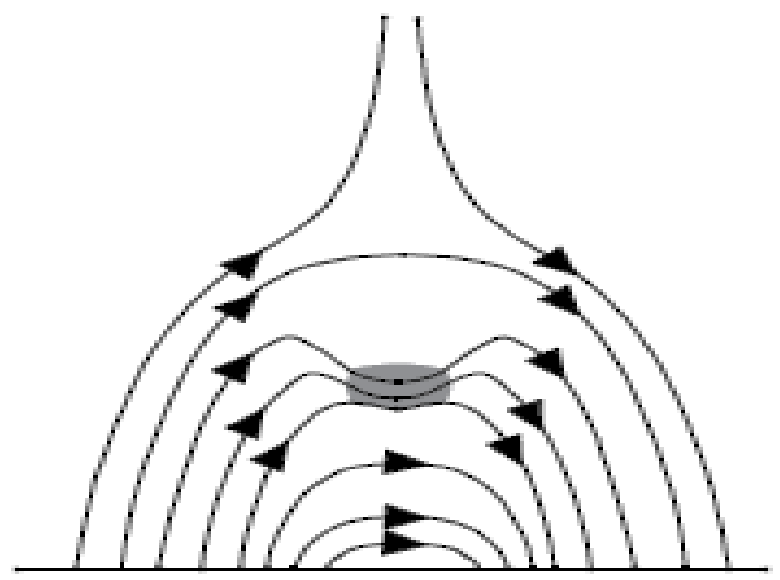

Figure 7: Magnetic topology in Kippenhahn-Schluter model [72]

Kippenhahn and Schluter [71] gave the simplest model of the internal structure of a prominence in which prominence was a simple arcade-like structure above the photosphere (see figure 7). In their model, magnetic tension provides the support for high-density plasma against the gravity. Further, they suggested that there is high magnetic pressure away from the centre of the prominence. Due to this reason, plasma is compressed against the outward pressure gradient because of the magnetic pressure acting towards the centre. Prominences with different topologies have also been observed. Prominence with helical topologies have been studied in detail [73] - [78]. Observations also show that helical prominences frequently occur in active regions. Observations of eruptions of prominences displayed helical structure which is consistent with the idea of the helical magnetic field in prominence [74] - [78].

Raadu and Kuperus [79] studied the thermal instability of coronal neutral sheets and argued that the quiescent prominences are formed by thermal instabilities in coronal sheets. Further, in Kuperus and Raadu [80] gave a model in which they demonstrated that the magnetic structure in the coronal neutral sheets can help in the formation of the coronal magnetic field (see figure 8). They showed that there is no horizontal magnetic field to support prominence against gravity as shown in the model of Kippenhahn-Schluter [71] and argued that forces on current filaments embedded in the magnetically neutral sheets can support a prominence due to induced currents in the photospheric boundary. In their model, they concluded that magnetic field annihilation and reconnection are a major phenomenon for stability and support of prominence. They further concluded that dis-partition brusque (sudden eruption of prominence) is the consequence of mass loss of prominence or large-scale changes in the photospheric field.

In Kippenhahn-Schluter (K-S) model, prominence has a simple arcade-like structure with normal magnetic polarity while in Kuperus-Raadu (K-R) model, the magnetic field had inverse polarity i.e., the magnetic field in prominence has opposite direction to that of the direction of magnetic field lines in the photosphere (see figure 8) [82].
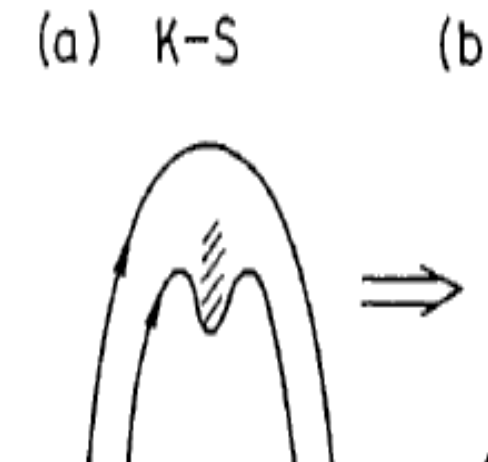

Figure 8: Evolutioin from Kippenhahn-Schluter $(K-S)$ model to Kuperus-Raadu $(K-R)$ model [81] (d) $K-R$
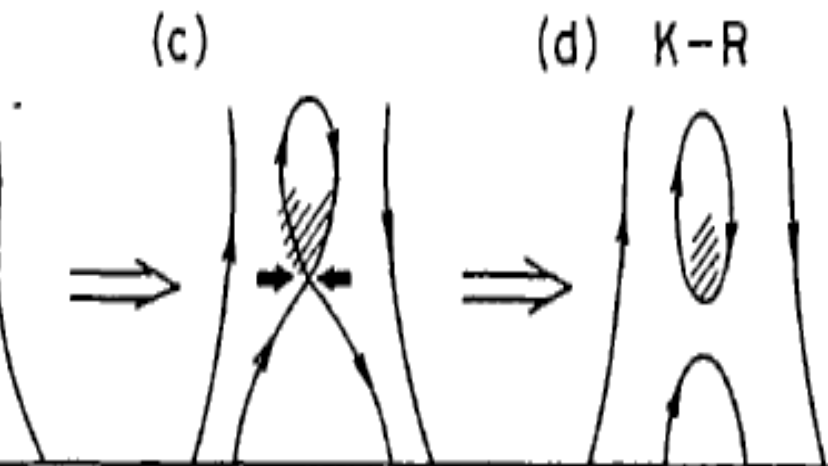
Solar Dynamical Processes I

Prominence eruption plays a very crucial role in solar energetic particle events. Their eruption can lead to coronal mass ejections which are further followed by a solar flare [83] - [86]. Magnetic reconnections and prominence interactions do play a very significant role in prominence stability. Magnetic reconnection in corona can lead to loop prominence phenomenon [85].

Prominences are closely related to various solar dynamical processes such as flares, CMEs etc. Prominence eruption mostly leads to a CME which affect the interplanetary space very drastically and affect interplanetary space weather [87]. The particles and radiations emitted during the eruption can reach Earth and may lead to the various phenomenon on Earth. Sometimes, it provides fascinating views like auroras at the poles while sometimes it can lead to power grid failure, communication system, radar system failure and radiation danger on humans.

\subsection{Bright points}

Bright points (hereafter BPs) are one of the most observed and widespread dynamical processes on Sun. Depending upon the wavelength of observation, they are called X-ray bright points (XBPs) or EUV bright points. They were first noted by the analysis of X-ray images from Skylab in 1970. They were called "bright point-like X-ray features" by Krieger et. al. [88]. A BP is often identified by small-scale bright flash above the photosphere. Golub [89] defined "XBPs as a closed magnetically bipolar region of soft X-ray emission that is less than 1' in diameter". They are also called coronal bright points (CBPs). A study of intensity oscillation of XBP reveals that their intensity can be tens of times brighter than the surrounding corona [90]. From their discovery, BPs have been studied in detail and Table 3 shows few of their approximate properties found by the analysis of X-ray images:

Table 3: Properties of bright points, [91] - [93]

\begin{tabular}{|l|c|}
\hline Property & Value (average) \\
\hline Temperature & $1.3-1.6 \mathrm{MK}$ \\
\hline Magnetic Field & 10 Gauss \\
\hline Area & $10^{14} \mathrm{~m}^{2}$ \\
\hline Ascent speed & $2 \mathrm{~km} / \mathrm{s}$ \\
\hline Size & $10^{4} \mathrm{~km}$ \\
\hline Density & $10^{9} \mathrm{~cm}^{-3}$ \\
\hline
\end{tabular}

Golub et. al. [92] suggested that on an average, around 1500 BPs emerge out per day all over the Sun, bring out approximately $10^{22} \mathrm{Mx}$ of magnetic flux. They also estimated that the average lifetime of a BP is about 8 hours. However, on the basis of data obtained from the New Solar Telescope (NST), Abramenko et al. [94] reported that around $98.6 \%$ of BPs had a lifetime of fewer than 2 minutes. In their recent work, Alipour et al. [95] suggested that around $52 \%$ of all CBP lived for less than 20 min while remaining had an average lifetime of about 6 hours. It has also been observed that BPs and sunspot number are anticorrelated [95], [96]. Early inspection of BPs variation over a solar cycle suggested that number of BPs increase during solar minimum [97] - [98] while Hara et. al., [99] suggested that there is no increase in BP number during solar minimum. They also suggested that solar activity cycle and BPs are nearly independent of each other.

An early investigation from various observations [88], [89], [92], [93] suggested that BPs are the region of the bipolar magnetic field which helps to emerge magnetic flux into the corona. It was hypothesized by Golub et. al. [92] that the turbulence in the convection zone can lead to enhancement of subsurface field which can help in the development of BPs. In 1979, Sheeley and Golub [100] analyzed fine structure of CBPs and found that BPs consist of the rapidly evolving small loop-like structures.

Various models, suggesting a relationship of magnetic energy dissipation in BPs with the heating of corona and other coronal structures has been suggested [101] - [108]. In 1979, J.H. Piddington [101] suggested a magnetic loop and bubble model for XBP. In this model, kink or helical instability in twisted flux ropes 
Mishra et al., Adv. J. Grad. Res.; Vol. 3 Issue 1, pp: 47-61, January 2018

helps in the formation of the loop. Formed loop (magnetic bubble) rises into the photosphere due to buoyancy, upheaving the immersed loops out the sun and contributing a significant amount of magnetic energy and matter into the atmosphere of the Sun. He also suggested that to meet the energy requirement, XBPs must contain three or more flux fibres. This conclusion of Piddington is consistent with the observational analysis of Sheeley et al. [100].

In 1993, Webb et. al. [102] at Big Bear Solar Observatory studied the BPs and their relationship with magnetic features using X-ray and magnetogram data. They found that most of the XBPs were correlated to converging opposite polarity magnetic features. They further suggested that converging magnetic features of opposite polarity may undergo reconnection due to the cancellation of flux which can give rise to XBPs. A 2-dimensional model of converging flux at BP, inspired by the discovery of cancelling magnetic feature at Big Bear Observatory, was given by Priest et al [105]. They suggested in their model that XBPs are the manifestation of the dynamic behaviour of magnetic fragments in three stage processes (see figure 9): a pre-interaction phase, an interaction phase and a cancellation phase. In pre-interaction phase, magnetic fragments of opposite polarity from different sources move towards each other and form a null point in the photosphere. In the interaction phase, null point evolves into an X-point releasing magnetic energy and starts rising into the corona which further leads to the creation of XBPs accompanied by jets. In the cancellation phase, magnetic features from strong sources cancel the magnetic features from weaker sources. Parnell et al. [100] used the converging flux model by priest et al. [99] to the model 3-dimensional structure of XBPs observed using NIXT [101].
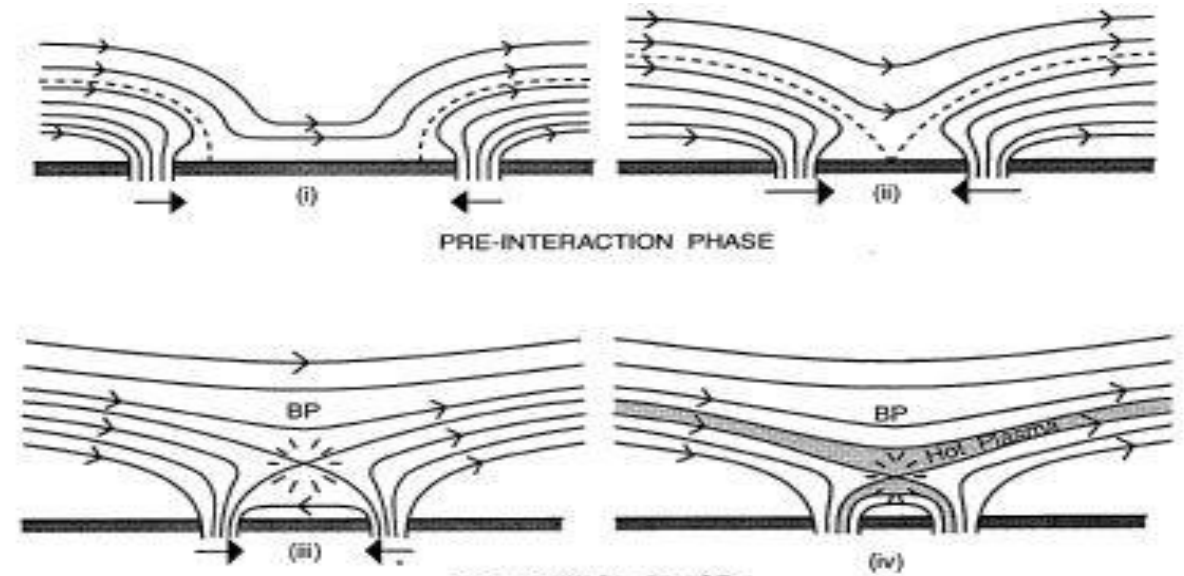

INTEAACTION PHASE

(w)
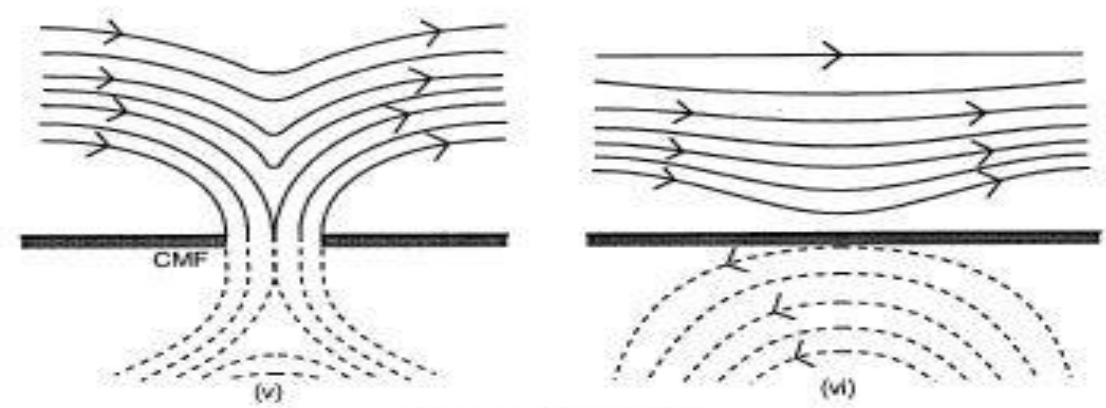

(vi)

CANCELLATION PHASE

Figure 9: Three phases in flux convergence model, (i) magnetic fragments approach each other, (ii) null point is formed, (iii) - (iv) formation of XBP, (v) cancellation of magnetic fragments in the photosphere, (vi) separation of magnetic flux above and below the photosphere [105]

In 1998, a 3-dimensional model for the appearance of BPs was given by D. W. Longscope [108] suggesting that before the interaction, magnetic fragments come closer to each other and form field line known as a separator. He further suggested that it is the magnetic reconnection at the separator where magnetic energy is dissipated and this, in turn, helps to form XBPs. 
Solar Dynamical Processes I

Observations from soft X-ray telescope on Yohkoh satellite [109] revealed the presence of X-ray jets in the corona. It was found that flares in XBPs were correlated to X-ray jets and reconnection processes played a very important role in their origin [110]. A recent study by Pucci et al [111] also revealed that X-ray jets have a close correspondence with XBPs. Analysis of various observational data of XBPs has revealed the presence of oscillations/periodic brightening in XBPs [90], [112], [113] .

It is now well established that magnetic reconnection plays a major role in the creation of BPs. They have been linked to flares and their properties have been studied in detail to better understand the onset of flares.

\section{Conclusion}

We have reviewed various dynamical processes taking place on Sun. We found that the magnetic interactions are the sole sources of these processes. We also tried to develop the basic understanding of scattered and uneven magnetic field on Sun through the differential rotation and dynamo model. In this article, we emphasized on the factors responsible for solar dynamical processes and briefly reviewed dynamical processes such as sunspot, prominence and bright points. Sunspots are cool, dark regions on the photosphere with very high magnetic field strength. They travel from equator to pole during their 11year old cycle and hence defining solar maxima and minima. Sunspot motion along PIL was found helpful in the flux tube rise and magnetic reconnection. These magnetic reconnections play a fundamental role in various other dynamical processes such as prominences and bright points. We reviewed various prominence models explaining various observational properties. Prominence can have many kinds of a structure such as a simple arch-like structure or a complex helical magnetic flux tube structure depending on various factors such as location on the photosphere, surrounding magnetic field, solar cycle (maxima or minima) etc. Prominence eruption can trigger various dynamical processes such as coronal mass ejection (CME) and solar flare. We also presented a brief account of bright points (BPs) which mainly results from flux emergence and magnetic reconnection. BPs are considered to be the site of magnetic reconnection on the solar surface and can be helpful in the understanding other dynamical processes such as solar flares, solar jets etc.

\section{How to Cite this Article:}

A. Mishra and M. Kumar "Solar Dynamical Processes I", Advanced Journal of Graduate Research, vol. 3, no. 1, pp. 47-61, Jan. 2018. doi: 10.21467/ajgr.3.1. 47-61

\section{References}

[1] B.N. Dwivedi, E.N. Parker, Dynamic Sun, Cambridge University Press ISBN - 9780521810579

[2] D.N. Baker, "Introduction to space weather", Lect. Notes Phys. 656, 3-20, 2005.

[3] M. Moldwin, "Introduction to space weather", 2008, ISBN - 9780521711128

[4] M. Schussler, "The Sun and Its Restless Magnetic Field”, Lect. Notes Phys. 656, 23-49, 2005.

[5] K.J.H. Phillips, "Guide to the Sun", Cambridge University Press, ISBN - 978052139788

[6] J.-P. Rozelot, C. Neiner (eds.), The Environments of the Sun and the Stars, Lecture Notes in Physics 857, 2013.

[7] J. -P. Rozelot, Solar and Heliospheric Origins of Space Weather Phenomena, Lect. Notes Phys. 699.

[8] J.P. Rozelot and S. Lefebvre, "Advances in Understanding Elements of the Sun-Earth Links", Solar and Heliospheric Origins of Space Weather Phenomena, Lect. Notes Phys. 699, 5-24, 2006. :

[9] Dina Prialnik, "An introduction to the theory of stellar structure and evolution", Cambridge University Press, ISBN - 9780521866040

[10] A.G.W. Cameron, "Abundances of the elements in the solar system”, Space Science Reviews, 15, 121-146, 1973.

[11] M. Asplund, N. Grevesse, A.J. Sauval, P. Scott, "The chemical coomposition of the Sun”, arXiv: 0909.0948v1, 2009.

[12] S. Turck-Chieze, S. Couvidat, "Solar neutrinos, helioseismology and the solar internal dynamics", arXiv:1009.0852v2 [astro-ph.SR], 2011.

[13] A. Ianni, "Solar neutrinos and the solar model", Physics of the Dark universe, 4, 44-49, 2014.

[14] G.D. Orebi Gann, "Everything under the Sun: A review of solar neutrinos", arXiv:1504.02154v2 [nucl-ex], 2015.

[15] S. Basu, "Global seismology of the Sun", arXiv: 1606.07071v1 [astro-ph.SR], 2016.

[16] T. Sakurai, "Helioseismology, dynamo and magnetic helicity", The 61st Fujihara Seminar: Progress in Solar / Stellar Physics with Helio- and Asteroseismology, ASP Conference Series, Vol. 462, H. Shibahashi, M. Takata, and A. E. Lynas-Gray, eds., 2012.

[17] S.M. Chitre, H.M. Antia, "Seismic Sun", From the book Dynamic Sun edited by B.N. Dwivedi, foreword by E.N. Parker, Cambridge University Press ISBN - 9780521810579

[18] G. Vigeesh, PhD Theses, Indian Institute of Astrophysics, 2010. 
Mishra et al., Adv. J. Grad. Res.; Vol. 3 Issue 1, pp: 47-61, January 2018

[19] H.J. Haubold, A.M. Mathai, "Solar nuclear energy generation and the chlorine solar neutrino experiment", arXiv:9405040v1 [astroph], 1994.

[20] J.C. Vial, "Nature and variability of plasma ejected by the Sun", J.-P Rozelot, C. Neiner (eds), The Environment of the Sun and the Stars, Lecture Notes in Physics 857.

[21] A. Nordlund, "Solar photosphere and convection", From the book Dynamic Sun edited by B.N. Dwivedi, foreword by E.N. Parker, Cambridge University Press ISBN - 9780521810579

[22] P. Ulmschneider, W. Kalkofen, "Heating of solar chromosphere", From the book Dynamic Sun edited by B.N. Dwivedi, foreword by E.N. Parker, Cambridge University Press ISBN - 9780521810579

[23] T. Wiegelmann, S.K. Solanki, "Why are coronal holes distinguishable from the quiet Sun in transition region radiation?", Proceedings of the SOHO 15 Workshop-Coronal heating, 2004.

[24] H.W. Babcock, H.D. Babcock, “The Sun's magnetic field, 1952 -1954”, The Astrophysical Journal, 121, 349, 1955.

[25] H.W. Babcock, "The topology of the Sun's magnetic field and the 22-year cycle", The Astrophysical Journal, 133, $572,1961$.

[26] H.W. Babcock, T.G. Cowling, "General magnetic fields in the Sun and stars", Mon. Not. R. Astr. Soc., 113, 357, 1953.

[27] J. W. Dungey, "The motion of magnetic fields", MNRAS, 113, 1953.

[28] R.B. Leighton, 1964, "Transport of magnetic fields on the Sun”, The Astrophysical Journal, 140, 1547, 1964.

[29] R.B. Leighton, 1969, "A magneto-kinematic model of the solar cycle", The Astrophysical Journal, 156, 1969.

[30] T. Wiegelmann, T. Sakurai, "Solar force free magnetic field", arXiv: 1208.4693v1 [astro-ph.SR], 2012.

[31] H. Zirin, "Fine Structure of Solar Magnetic Field", Solar Physics, 22, 34-48, 1972.

[32] A.R. Choudhari, "The solar dynamo as a model of the solar cycle", From the book Dynamic Sun, Edited by B.N. Dwivedi, Foreword by E.N. Parker, Cambridge University Press ISBN - 9780521810579

[33] A.R. Choudhuri, "The elementary introduction to solar dynamo theory", 2007.

[34] P. Charbonneau, "Dynamo models of the solar cycle", Living Reviews in Solar Physics, 7, 2010.

[35] K. Georgieva, "Space weather and Space Climate - What the Look from the Earth Tells Us About the Sun", J.-P Rozelot, C.Neiner (eds), The Environment of The Sun and The Stars, Lecture notes in Physics, 857, 2013.

[36] L.L. Kichatinov, "Origin of the Sun's differential rotation", AUS, 138, 1990.

[37] E.N. Parker, "Hydromagnetic Dynamo Models", The Astrophysical Journal, 122, 293, 1955.

[38] Spiro K. Antiochos, "Solar Drivers of Space Weather", ASP Conference Series, Volume 95, 1996.

[39] J.M. Vaquero, "Historical sunspot observations: A Review", 2007.

[40] R.C. Carrington, "On the evidence which the observed motions of the solar spots offer for the existence of an atmosphere surrounding the Sun", MNRAS, 18, 169-177, 1858.

[41] R.C. Carrington, "On certain phenomena in the motions of solar spots", MNRAS, 19, 81-84, 1859.

[42] G.E. Hale, "On the probable existence of a magnetic field in Sunspot", The Astrophysical Journal, 28, 315, 1908.

[43] G.E. Hale, F. Ellerman, S.B. Nicholson, A.H. Joy, 1919, "The Magnetic polarity of sunspots", The Astrophysical Journal, 49, 153, 1919.

[44] T. G. Cowling, "The growth and decay of the sunspot magnetic field", MNRAS, 106, 1946.

[45] H. Alfven, "On the theory of sunspots", 1956.

[46] F. Meyer, H.U. Schmidt, N.O. Weiss, P.R. Wilson, "The growth and decay of Sunspots", Mon. Not. R. Astr. Soc., 169, 35-57, 1974.

[47] S.K. Solanki, "Sunspots: A Review", The Astronomy and Astrophysics Review, 11, 153-286, 2003.

[48] K. Ichimoto, Y. Suematsu, S. Tsuneta, Y. Katsukawa, T. Shimizu, R.A. Shine, T.D. Tarbell, A.M. Title, B.W. Lites, M. Kubo, S. Nagata, "Twisting motions of sunspot penumbral filaments", Science. 318, 2007.

[49] J.M. Borrero, K. Ichimoto, "Magnetic structure of sunspots", Living reviews Solar physics, 8, 2011.

[50] B. Geerts, E. Linacre, "Sunspots and Climate", 1977.

[51] C. de Jager, "Solar activity and its influence on climate", Netherland Journal of Geosciences, 87-3, 207-213, 2008.

[52] B. Kirov, S. Asenovski, K. Georgieva, V.N. Obridka, "What causes geomagnetic activity during sunspot minimum?", Солнечная и солнечно-земная физика, Санкт-Петербург, Пулково, 20 - 24 октября, 2014.

[53] A. Raja Bayanna, Shibu K. Mathew, Brajesh Kumar, Rohan E. Louis, P Venkatakrishnan, "A study of the relation between intensity oscillations and magnetic field parameters in a sunspot: Hinode Observations", arXiv:1407.7363v1 [astro-ph: SR], 2014.

[54] Robert Sych, "MHD waves in sunspots", arXiv: 1509.06466v1 [astro-ph: SR], 2015.

[55] R. Sych, M. Karlicky, A. Altyntsev, J. Dudik, L. Kashapova, "Sunspot waves and flare energy release", Astronomy and Astrophysics, 577, A43, 2015.

[56] Roberto Soler, Jaume Terradas, Ramon Oliver, Jose Luis Ballaster, "The role of Alfven wave heating in solar prominences", Astronomy and Astrophysics, 592, A28, 2016.

[57] E. Pettit, "The properties of solar prominences as related to type", The Astrophysical Journal, 98, 6-19, 1943.

[58] S.K. Antiochos, P.J. MacNeice, D.S. Spicer, J.A. Klimchuk, "The dynamic formation of prominence condensations", The Astrophysical Journal, 512, 985-991, 1999.

[59] B.C. Low, B. Fong, Y. Fan, "The mass of a solar quiescent prominence”, The Astrophysical Journal, 594, 1060-1067, 2003.

[60] B.C. Low, "Solar Activity and the Corona", Solar Physics, 167: 217-265, 1996.

[61] D.M. Rust, "Magnetic fields in quiescent solar prominences. I. Observations", The Astrophysical Journal, 150, 313-326, 1967.

[62] D.M. Rust, "Magnetic fields in quiescent solar prominences. II. Photospheric sources", The Astrophysical Journal, 160, 315-324, 1970 .

[63] M. Correll, W.O. Roberts, "Atmospheric magnetic fields above active solar region of April 13, 1950”, The Astrophysical Journal, 127, 726-730, 1958. 
Solar Dynamical Processes I

[64] H. Zirin, A. Severny, "Measurement of magnetic fields in solar prominences", Obs, 81, 155-156, 1961.

[65] C. Kuckein, R. Centeno, V.M. Pillet, R. Casini, R.M. Sainz, T. Shimizu, "Magnetic field strength of active region filaments", Astronomy and Astrophysics, 501, 1113-1121, 2009.

[66] J.L. Leroy, V. Bommier, S. Sahal-Brechot, "The magnetic field in the prominences of the polar crown", Solar Physics, 83, 135-142, 1983.

[67] G. Zirin, "Magnetic field in solar prominences", Soviet Astronomy - AJ, Vol.5, No.5, 660-666, 1962.

[68] D.H. Mackay, J.T. Karpen, J.L. Ballester, B. Schmieder, G. Aulanier, "Physics of solar prominences: II - Magnetic structure and dynamics", arXiv:1001.1635.v1, 2010.

[69] P.I. Papics, "Solar prominence, solar flares and coronal mass ejection: A solar physics report", 2007.

[70] S. Parenti, "Solar prominence: Observations", Living Reviews in Solar Physics, 11, 1, 2014.

[71] R. Kippenhahn, A. Schluter, "Eine Theorie der Solaren Filamente, Zeitschrift fur Astrophysik", 43, 36-62, 1957.

[72] H.R. Gilbert, T.E. Holzer, B.C. Low, J.T. Burkepile, "Observational interpretation of an active prominence on 1999 May 1", The Astrophysical Journal, 549, 1221-1230, 2001.

[73] U. Anzer, E. Tandberg-Hanssen, "A model for quiescent prominences with helical structure”, Solar Physics, 11, 61-67, 1970.

[74] B. Vrsnak, V. Ruzdjak, R. Brajsa, A. Dzubur, "Structure and stability of prominences with helical structure”, Solar Physics, 116, 4560, 1988.

[75] B. Vrsnak, V. Ruzdjak, B. Rompolt, "Stability of prominences exposing helical-like patterns”, Solar Physics, 136, 151-167, 1991.

[76] D.H. Mackay, V. Gaizauskas, "Helicity as a component of filament formation”, Solar physics, 216, 121-142, 2003.

[77] T.J. Okamoto, S. Tsuneta, B.W.Lites, M. Kubo, T. Yokoyama, T.E. Berger, K. Ichimoto, Y. Katsukawa, S. Nagata, K. Shibata, T. Shimizu, R.A. Shine, Y. Suematsu, T.D. Tarbell, A.M. Title, "Prominence formation associated with an emerging helical flux rope", arXiv:0904.0007v1, 2009.

[78] D.M. Rust, A. Kumar, "Helical magnetic fields in filaments", Solar Physics, 155, 69-97, 1994.

[79] M.A. Raadu, M. Kuperus, "Thermal instability of coronal neutral sheets and the formation of quiescent prominence", Solar Physics, 28, 77-94, 1973.

[80] M. Kuperus, M.A. Raadu, "The support of prominence formed in neutral sheets", Astronomy and Astrophysics, 31, 189-193, 1974.

[81] T. Hirayama, "Modern observations of solar prominences", Solar Physics, 100, 415, 1985.

[82] E.R., Priest, A.W. Hood, U. Anzer, "A twisted flux tube model for solar prominences I. General properties", The Astrophysical Journal, 344, 1010-1025, 1989.

[83] S.W. Kahler, E.W. Cliver, H.V. Cane, R.E. McGuire, R.G. Stone, N.R. Sheeley Jr., "Solar filament eruptions and energetic particle events", The Astrophysical Journal, 302: 504-510, 1986.

[84] S.W. Kahler, R.L. Moore, S.R. Kane, H. Zirin, "Filament eruptions and the impulsive phase of solar flares", The Astrophysical Journal, 328, 824-829, 1988

[85] R.A. Kopp, G.W. Pneuman, "Magnetic reconnection in the corona and the loop prominence phenomenon", Solar Physics, 50, 85-98, 1976.

[86] G.E. Moreton, H.E. Ramsey, "Recent observations of dynamical phenomena associated with solar flares", PASP, 72, 1960.

[87] A Mishra and M Kumar "An insight into Space Weather", Advanced Journal of Graduate Research, vol. 2, no. 1, pp. 46-57, 2017.

[88] A.S. Krieger, G.S. Vaiana, L.P. Van Speybroec, "The x-ray corona and the photospheric magnetic field”, IAUS, 43, $397,1971$.

[89] L. Golub, "X-ray bright points and the solar cycle”, Phil. Trans. R. Soc. Lond. A, 297, 595-604, 1980.

[90] R. Kariyappa, B.A. Varghese, "Intensity oscillations and heating of the coronal X-ray bright points from Hinode/XRT", Astronomy and Astrophysics, 485, 289-292, 2008.

[91] D.S. Brown, C.E. Parnell, E.E. DeLuca, R.A. Mcmullen, L. Golub, E.R. Priest, "Magnetic structure and reconnection of X-ray bright points in the solar corona", Adv. Space Rev., Vol. 29, No. 7, 1093-1099, 2002.

[92] L. Golub, A.S. Krieger, J.K. Silk, A.F. Timothy, G.S. Vaiana, "Solar X-ray bright points", The Astrophysical Journal, 189, L93-L97, 1974.

[93] L. Golub, A.S. Krieger, J.K. Silk, J.W. Harvey, G.S. Vaiana, "Magnetic properties of X-ray bright points”, Solar Physics, 53, 111121,1977

[94] V. Abramenko, V. Yurchyshyn, Philip Goode, Ali Kilcik, "Statistical Distribution of size and lifetime of bright points observed with the New Solar Telescope", arXiv: 1012.1584v1 [astro-ph.SR], 2010.

[95] N. Alipour, H. Safari, "Statistical properties of solar coronal bright points", The Astrophysical Journal, 807, 175, (9pp), 2015.

[96] L. Golub, J.M. Davis, A.S. Krieger, “Anticorrelation of X-ray bright points with sunspot number, 1970 - 1998”, The Astrophysical Journal, 229, L145-L1590, 1979.

[97] J. M. Davis, "X-ray bright points and the solar cycle dependence of emerging magnetic flux", NASCP, 2098, 65,1980.

[98] L. Golub, G.S. Vaiana, "X-ray bright points and the solar cycle", NASCP, 2098, 75, 1980.

[99] H. Hara, K. Nakakubo-Morimoto, "Variation of the X-ray bright point number over the solar activity cycle", The Astrophysical Journal, 589, 1062-1074, 2003.

[100] N.R. Sheeley Jr., L. Golub, "Rapid changes in the fine structure of a coronal bright point and a small coronal active region", Solar Physics, 63, 119-126, 1979.

[101] J.H. Piddington, “A model for solar X-ray bright points and ephemeral active regions", Aust. J. Phys., 32, 671-80, 1979.:

[102] D.F. Webb, S.F. Martin, D. Moses, J.W. Harvey, "The correspondence between X-ray bright points and evolving magnetic features in the quiet sun", Solar physics, 144, 15-35, 1993

[103] W.M. Glencross, "Heating of coronal material at X-ray bright points", The Astrophysical Journal, 199, L53-L56, 1975.

[104] M.H. Gokhale, "X-ray bright points, coronal heating and the solar cycle", Solar Physics, 41, 381-386, 1975. 
Mishra et al., Adv. J. Grad. Res.; Vol. 3 Issue 1, pp: 47-61, January 2018

[105] E.R. Priest, C.E. Parnell, S.F. Martin, "A converging flux model of an X-ray bright point and an associated cancelling magnetic feature", The Astrophysical Journal, 427, 459-474, 1994.

[106] C.E. Parnell, E.R. Priest, L.Golub, "The three-dimensional structures of X-ray bright points", Solar Physics, 151, 57-74, 1994.

[107] C.E. Parnell, E.R. Priest, V.S. Titov, “A model for X-ray bright points due to unequal cancelling flux sources”, Solar Physics, 153, 217-235, 1994.

[108] D.W. Longscope, “A model for current sheets and reconnection in X-ray bright points", The Astrophysical Journal, 507, 433-442, 1998.

[109] K. Shibata, Y. Ishido, L.W. Acton, K.T. Strong, T. Hirayama, Y. Uchida, A.H. McAllister, R. Matsumoto, S. Tsuneta, T. Shimizu, H. Hara, T. Sakurai, K. Ichimoto, Y. Nischino, Y. Ogawara, "Observations of X-ray jets with the Yohkoh Soft X-ray Telescope", PASJ, 44, L173-L179, 1992.

[110] T. Yokoyama, K Shibata, "Numerical simulation of solar coronal X-ray jets based on the magnetic reconnection model", PASJ, 48, 353-376, 1996.

[111] S. Pucci, G. Poletto, A.C. Sterling, M. Romoli, "Solar Polar X-ray jets and multiple bright points: Evidence for sympathetic activity", The Astrophysical Journal Letters, 745, L31 (5pp), 2012.

[112] M. Kumar, A. K. Srivastava, B.N. Dwivedi, "Observation of intensity oscillations above X-ray bright points from the Hinode/XRT: Signature of magnetohydrodynamic oscillations in the solar corona", MNRAS, 415, 1419-1425, 2011.

[113] T. Samanta, D. Banerjee, H. Tian, "Quasi-periodic oscillation of a coronal bright point”, The Astrophysical Journal, 806, 172 (6pp), 2015.

Publish your research article in AIJR journals-

$\checkmark \quad$ Online Submission and Tracking

$\checkmark$ Peer-Reviewed

$\checkmark \quad$ Rapid decision

$\checkmark \quad$ Immediate Publication after acceptance

$\checkmark \quad$ Articles freely available online

$\checkmark \quad$ Retain full copyright of your article.

Submit your article at journals.aijr.in
Publish your books with AIJR publisher-

$\checkmark$ Publish with ISBN and DOI.

$\checkmark$ Publish Thesis/Dissertation as Monograph.

$\checkmark$ Publish Book Monograph.

$\checkmark$ Publish Edited Volume/ Book.

$\checkmark$ Publish Conference Proceedings

$\checkmark \quad$ Retain full copyright of your books.

Submit your manuscript at books.aijr.org- 